\title{
CoBuilt
}

\section{Towards a novel methodology for workflow capture and analysis of carpentry tasks for human-robot collaboration}

\author{
Dagmar Reinhardt ${ }^{1}$, M. Hank Haeusler ${ }^{2}$, Lian Loke , $^{3}$ \\ Eduardo de Oliveira Barata ${ }^{4}$, Charlotte Firth ${ }^{5}$, Nariddh Khean ${ }^{6}$, \\ Kerry London ${ }^{7}$, Yingbin Feng ${ }^{8}$, Rodney Watt $^{9}$ \\ 1,3,4,9 University of Sydney ${ }^{2}$ UNSW Computational Design / CAFA Visual Innovation \\ Institute Beijing ${ }^{5,6}$ UNSW / Computational Design ${ }^{7,8}$ Western Sydney University \\ 1,3,4,9 \{dagmar.reinhardt|lian.loke|eduardo.barata|rodney.watt\}@sydney. \\ edu.au ${ }^{2,5,6}\{$ m.haeusler|c.firth|n.khean\}@unsw.edu.au \\ ${ }^{7,8}\{$ K.London|Y.Feng\}@westernsydney.edu.au
}

\begin{abstract}
Advanced manufacturing and robotic fabrication for the housing construction industry is mainly focused on the use of industrial robots in the pre-fabrication stage. Yet to be fully developed is the use on-site of collaborative robots, able to work cooperatively with humans in a range of construction trades. Our study focuses on the trade of carpentry in small-to-medium size enterprises in the Australian construction industry, seeking to understand and identify opportunities in the current workflows of carpenters for the role of collaborative robots. Prior to presenting solutions for this problem, we first developed a novel methodology for the capture and analysis of the body movements of carpenters, resulting in a suite of visual resources to aid us in thinking through where, what, and how a collaborative robot could participate in the carpentry task. We report on the challenges involved, and outline how the results of applying this methodology will inform the next stage of our research.
\end{abstract}

Keywords: Robotic Fabrication, Collaborative Robots, Training Methodology, Machine Learning, Interaction Analysis

\section{INTRODUCTION}

According to the Plan for Growing Sydney, New South Wales will need to accommodate 1.6 million new residents over the next 20 years (NSW Government, 2014). In New South Wales, increasing shortfall of building tradespersons, such as bricklayers, carpenters and stonemasons, will cause a lack of rapid response to this unprecedented growth, potentially resulting in the escalation of housing costs and construction times. This is not only a local phenomena but can be observed globally. In the 2018 online [5] article Robotics in Construction: A brief History of Automation in the Industry by Higgins the author points out that the construction industry is 
rife for an adoption of robotics into construction processes. Listing issues such as waste, labor productivity, and shortage of skilled labour, Higgins concludes that the construction industry is "in a technologyadopting period, with general robotics technologies being translated into specific use cases solving problems in construction" [5]. To prevent the cascade effect of escalation of housing costs and construction times, and to provide more affordable housing in areas close to friends and family, workplaces and schools, public transport, and shops and services, an innovative approach to the problem is required, involving the design of a unique, bespoke, and interactive relationship between human craftsmanship and machine intelligence via Cyber囚Physical Systems and Social Robotics.

Over the last decade, industrial robots from the automotive industry have been repurposed for use in material fabrication and built environment research in academic institutions around the globe. They are adaptable for a variety of construction techniques due to the versatility of their 6冈axis arm configuration. While their physical adaptability is an advantage, their computational and sensory capabilities are rudimentary and have not evolved significantly in the last century. Conversely, the manufacturing industry has moved on by introducing new means of production, namely Industry 4.0 (Rosenberg et al. 2015). In particular, Cyber囚Physical Systems are machines or mechanisms controlled or monitored by computer冈based algorithms that are tightly integrated with the Internet and its users. The U.S. Army Research Laboratory argues in 2018 that machine learning has advanced to the point where industrial robots are able to be trained to learn how to perform a task, instead of simply following a pre-programmed script [3]. Working closely with humans in shared environments, collaborative robots (CoBots) are capable of learning from demonstration to perform useful and repetitive tasks with optimised precision. The use of demonstration learning techniques for fabrication has recently found strong interest, which regarding to Technology Review in 2015 is particularly relevant when CoBots are used to work with material properties and performances for construction purposes [4]

The elaborated choreographies used in craft and trade have resulted from centuries in which practice methods and techniques were developed while adapting with the evolution of construction techniques and tooling processes. Consequently, movements of a collaborative robot are considered here not only for optimisation, efficiency, or safety, but to promote collaboration and interactions with humans. CoBots could be considered reliable co囚builders while depending on the human counterpart for responding to new challenges and situations with creativity and adaptability (Rosenberg et al. 2015).

\section{COBOTS-HUMAN ROBOT COLABORATION}

'Cobots' - also known as collaborative robots, corobots, and Intelligent Assist Devices (IADs)- are designed to allow humans and robots working together side-by-side, in direct physical interaction with a human user and within a shared workspace (Colgate and Peshkin, 1996). Human-Robot collaboration has generated a considerable amount of interest in robotics and manufacturing industries. Unlike traditional robot applications, collaborative applications allow operators to share their workspace safely. This benefits the manufacturing industry in terms of floor space utilisation, ergonomic improvement, productivity optimisation, and flexibility (Sylla 2017).

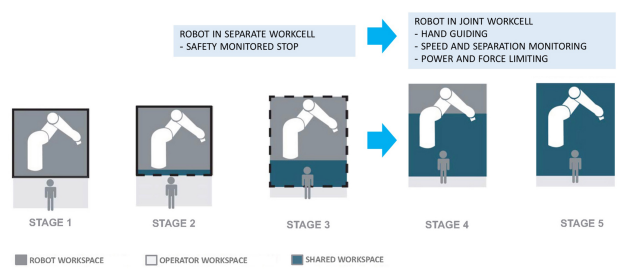

In principle, collaborative applications can be classified relative to level of workspace sharing; as full automation where an operator and robot workspace
Figure 1

Cobots - Human robot safe workspaces. Adopted from Staubli, 2017, Collaborative Robots: Performance vs Safety 
are separated by fencing, as cooperation in a shared workspace but tasks performed with a stationary robot, and as collaboration in a shared workspace and with shared task performance. Usually, employing advanced proximity sensing technology enables turning a conventional industrial robot into a cobot. Defined as International Standards ISO 10218 and to assist in co-working, collaborative features include safety monitored stops (where a cobot will cease movement temporarily when a human enters the workzone); hand guiding (for path teaching a robot through sequences of motions required to complete a task, using end effector technology to sense position and read forces); speed and separation monitoring (with frequent human intervention laser vision systems allow the robot to sense a human's proximity, and robot reactions can be programmed to perform at various distances between robot and human operator); and finally, power and force limiting (whereby robots read forces such as pressure, resistance or impacts using embedded sensors).

\section{RESEARCH SCOPE AND OBJECTIVES}

Developed as joint research between a government institution (Landcom) and academia (University of NSW, University of Sydney, and Western Sydney University), the larger research project aims to address the discrepancy between an unprecedented boom in urban growth combined with a severe shortage in skilled construction labour, while promoting an effective community engagement in redesigning construction and fabrication methodologies for cities. In order to achieve this goal, we first needed to develop a methodology for how to engage with construction workers, observe how they do their work, and analyse their current workflows for potential insertion of a CoBot, with particular attention applied to the use of movement, space, and time. This paper reports on the development for such a novel methodology that can be applied for capturing and analysing workflows from a choreographic (human-oriented) and kinematic (robot-focused) lens.

In the first, completed phase of this research, colleagues of Western Sydney University Construction Management department identified barriers and drivers to adoption of collaborative robotics to the construction industry, through workshops and semi冈structured interviews with Landcom's staff, and selected groups of housebuilders and subcontractors from various trades. This empirical analysis contributed to ground the findings from the literature review and helped to identify a specific trade and tasks within these trades that could be executed by collaborative robots at a latter phase in the project. Here, important contributions to the theoretical research field of construction work, health and safety were identified as well as the potential practical real『world benefits. It was important in this phase to recognise that whilst the major Australian developers (Tier 1) may be already looking at robotics, as seen in the case of Perth-based Fastbrick Robotics [6], it is not an area that appears to be much explored by SME (Small to Medium Enterprise) builders (Tier $2 \rrbracket 3$ ), and certainly not in collaborative robotics [7]. Amongst a broad spectrum of trades, such as masonry, stone冈masonry, plastering, glazing, and iron working, the trade of carpentry was identified as most suitable to determine the health and safety risks behind the repetition of a specific movement over a long period. Fig. 2 illustrates how each study phase contributes to the next.

\section{DEVELOPING A METHODOLOGY FOR HUMAN-ROBOT COLLABORATION}

In this section, we present the methodology developed in the context of carpentry, for empirical observations and analysis of the body movements of human workers, resulting in a suite of visual resources to aid us in thinking through where, what, and how a collaborative robot could participate in the carpentry task. Prior to running a live study on a construction site provided by our industry partner, Landcom, we ran a pilot study within The University of Sydney with in-house carpenters (Fig. 3). By doing the pilot study, we were able to develop and test the observe-andcapture steps of our proposed methodology, iron out 
STUDY 1

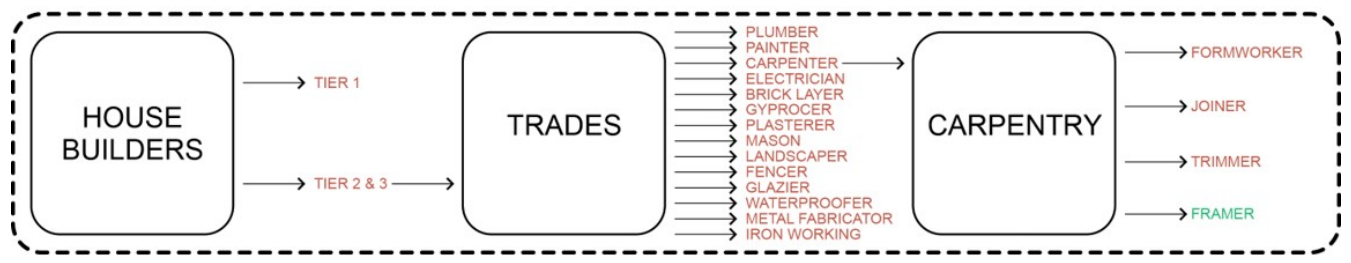

\section{STUDY 2}

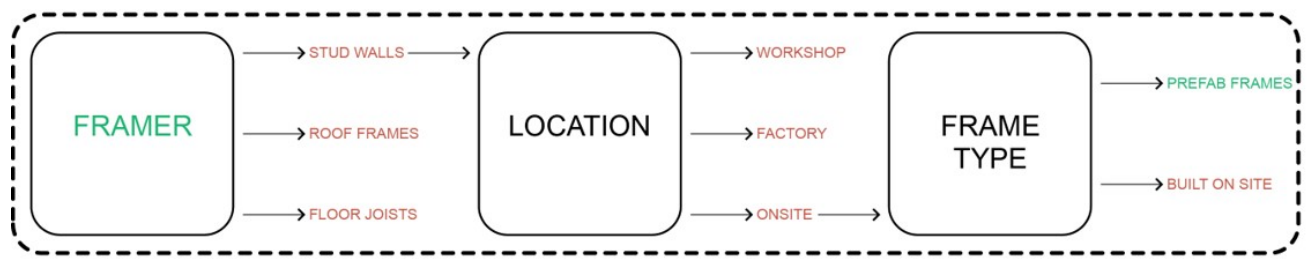

\section{STUDY 3}

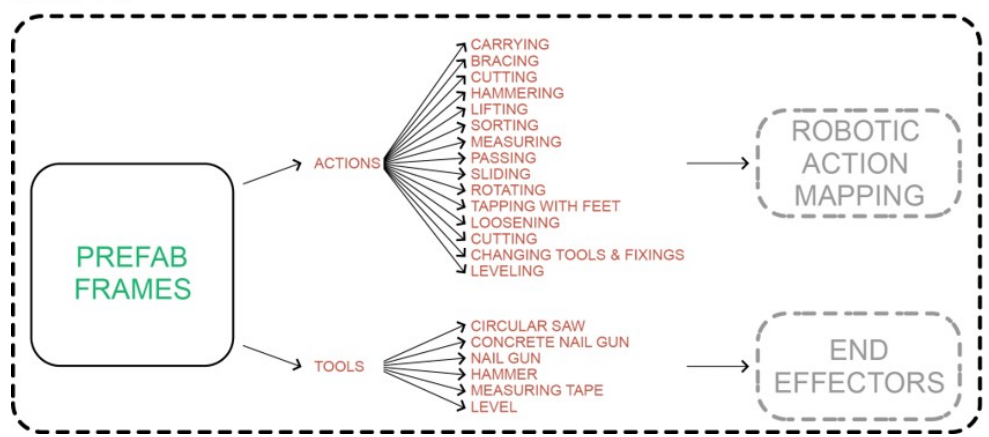

any process, logistical or equipment issues, and foresee any challenges on-site.

\section{Key Steps}

The key steps in our proposed methodology are:

1. Observe carpenters performing a typical task on a real construction site and capture this task in audio (dialogue) and video (action).

2. Analyse the video to identify

- Key Scenes
Figure 2

Study phase 1 to 3
- The set of actions performed by the carpenters

- The types of movements used to achieve the actions

- The use and relationship between person, tool and material

- The use of space by the carpenters

- Actions that required two people

- Rhythm and timing between two people
3. Transcribe the dialogue. The dialogue pro- 
Figure 3

Pilot Test Study in the Lab
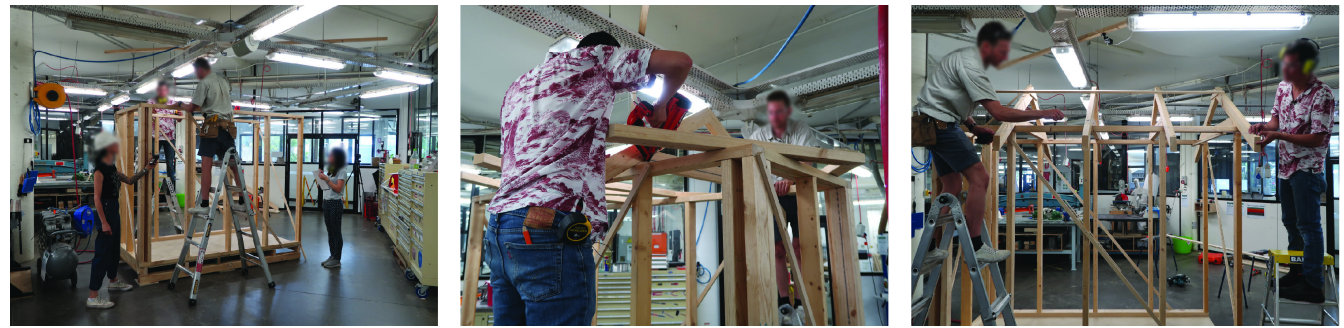

vides verbatim records of how the carpenters communicated to each other whilst performing tasks, and how they answered any questions posed by the researcher. Snippets of dialogue are highlighted, which provide insight into the why and how of performing physical tasks.

4. Generate a set of visual documentation from the above analysis to enable us to focus on different perspectives, relationships and information.

5. Identify potential parts of the workflow that are amenable to a collaborative robot working with a human carpenter, through inspection and evaluation of the visual documentation.

\section{Analysis of workflow}

The approach to analysis of the workflow (outlined in steps 2 and 3 ) is partly informed by the method of interaction analysis (Henderson and Jordan 1995), which provides a set of analytic foci to deconstruct a human activity in context. The foci of relevance to our study include activity, tool, material, environment, body, people, rhythm and timing, use of space, breakdowns and workarounds. Underlying the method of interaction analysis is an assumption that "knowledge and action are fundamentally social in origin, organization, and use, and are situated in particular social and material ecologies" (p.41, Henderson and Jordan 1995). The analysis seeks to "identify regularities in the ways in which participants utilize the resources of the complex social and material world of actors and objects in which they operate." (p.41, Henderson and Jordan 1995).

\section{The methodology in action}

Step 1. To explain and illustrate the application of our methodology, we use the selected case study of the framing process of carpentry that took place on a Landcom building site, where we had ethics approval and permission to record the activities of a team of carpenters constructing the structural walls of a single storey house from prefabricated timber structures. A single researcher with a Safe Work White Card conducted an empirical study on-site, taking video recordings of the activity and interviewing (audio recording) the carpenters whilst they were performing their tasks. When interviewing the carpenters, we were interested to learn why they were doing what they were doing, if what they were doing was normal, or a work-around, and any other factors that influenced the way they worked together.

Step 2 and 3. The video recordings (action) and audio recordings (dialogue) were imported into NVivo, a qualitative data analysis tool which allowed for the transcribing and segmenting of key motions, actions, collaboration and tool use during the framing process (See Table 1). NVivo is a qualitative data analysis package used for analysing big data sets. Time spans of each video file were captured from the timeline and overall descriptions given specific tasks being undertaken. Each time span was described in shorthand and coded into node categories. Nodes were organised under two separate lists - Physical Movements and Use of Tools. The node table describes a family of movements and operations over a 30 minute period.

By using NVivo these nodes were able to be 

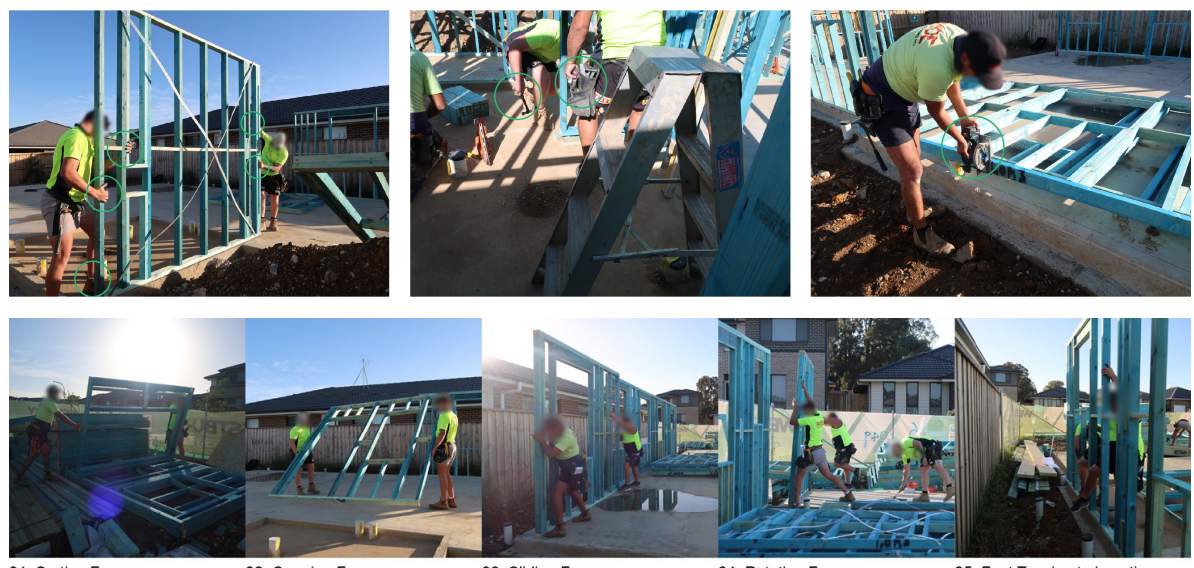

03. Sliding Frames

02. Carrying Frames

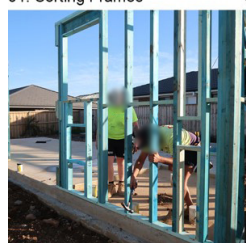

06. Hammer Tapping to Location 07 . Cutting for Doorways

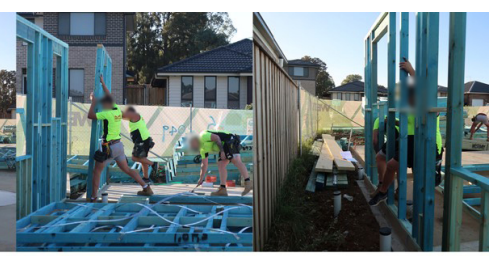

04. Rotating Frames
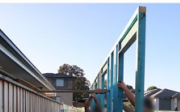

5. Foot Tapping to Locatio

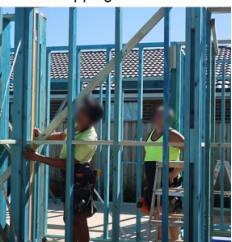

09. Temporary Supports

10. Levelling Frames

Nodes Table

\begin{tabular}{|c|c|c|c|c|c|}
\hline Physical Movements & Files & References & Use of Tools & Files & References \\
\hline Bracing & 1 & 1 & Circular Saw & 3 & 6 \\
\hline Carrying (1x Carpenter) & 1 & 1 & Concrete Nail Gun & 3 & 7 \\
\hline Carrying ( $2 \times$ Carpenter) & 3 & 16 & Hammer & 3 & 11 \\
\hline Changing Nail Gun Cartridge & 1 & 1 & Measuring Tape & 1 & 1 \\
\hline Cutting & 3 & 5 & Nail Gun (1x Carpenter) & 2 & 5 \\
\hline Hammering into Position & 3 & 5 & & & \\
\hline Lifting / Sorting & 2 & 5 & & & \\
\hline Loosening Straps & 3 & 5 & & & \\
\hline Measuring & 1 & 1 & & & \\
\hline Passing Tools & 1 & 1 & & & \\
\hline Position with Feet & 3 & 15 & & & \\
\hline Short Vertical Lift & 1 & 1 & & & \\
\hline Sliding Frame & 2 & 2 & & & \\
\hline Supporting in Position & 2 & 3 & & & \\
\hline Vertical Rotation & 3 & 14 & & & \\
\hline
\end{tabular}

Table 1

Node Table noting repetitive actions and use of tools

over 30 minutes.
Figure 4

Person-to-Material, Person-to-Tool and Person-to-Tool-\&Material

Figure 5

The series of photos show the 10 stage construction sequence involved in building the timber structure for a house from prefabricated frames on a live building site. 
Figure 6

Detailed framing actions highlighting how the carpenters use their hands and feet to help prop and stabilize the frames. extracted to demonstrate how often certain movements and tasks were being undertaken, and with what tools. Hierarchy diagrams could then be generated to describe where which movements or tools are used the most, and in which way. These general nodes can be understood as families, which will then have a subset of nodes categorising specific movements and all of their variations for interpretation.

Step 4. In presenting and explaining the results of the analysis, we are interested in what each visual resource enables us to see in the complex, messy reality of an active building site. In addition to NVivo a series of additional diagrams were generated to capture a number of relational views. These were outputted as person, tool and material. This documentation allows for the extraction of key information for each stage of the framing process, so that personto-material, person-to-tool and person-to-tool-andmaterial can be viewed as separate entities. This diagramatic process would then provide a resource for analysing potential positions for the Cobot to be placed. These diagrams typically show up to 10-15 stages of frame movement and final positioning.

We have selected key collaborative samples from a family of visualisations, as depicted below. The activities involving two carpenters working together to sort, lift, carry, slide, rotate, tap, hammer, cut, nail and level timber frames. The following series of annotated photographs highlights how the human body nimbly negotiates uneven terrain, uses a variety of limbs to support the frame in different positions, uses feet and tools to tap or nudge the structure into place.

The visual images capture and illustrate the spatial and interactional properties of the tasks, highlighting relationships between carpenters, structures, materials and tools. But what is not so obvious from the visual imagery are some of the temporal or sequencing-based information that is also of consequence in understanding the human activity. Some of this information can be found in the dialogue.

From the transcript of the talk between carpenters, we can see that even a seemingly simple task of securing a bracing material to a timber beam with a screw is in actuality a complex series of nuanced physical actions. In the example below of the head carpenter instructing the apprentice, he first of all tells him not to screw it in all the way, but to keep the screw somewhat loose to allow for some movement of the structure prior to the final tightening.

- Head carpenter: [0:03:18] yeah one there and there and on the bottom there. Yeah that's right. Don't tighten them with a drill. Just get em on hand tight - you know what I mean? Give me the drill.

- Other worker: [0:03:28] i'll just loosen em and take em and do them by hand

The transcript also revealed the types of dialogue related to the regular checking and confirming, so critical to the quality assurance of the construction process, that a material was in the correct position, or the appropriate tool was being used.

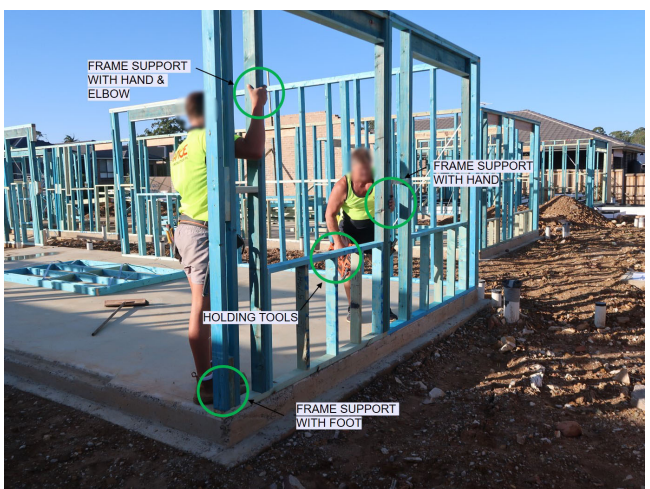

Step 5. Using the set of visual resources generated above, the final step involves an evaluation of how a cobot could assist or replace the human worker in the set of collaborative actions identified from the analysis. Table 2 contains a subset of the collaborative actions illustrated in Figure 6 (and listed in Table 1), identified for the activity of framing. In the evaluation, we assumed the cobot could have either one or two arms, and be either static or mobile. The degree 
of locomotion mobility and the number of arms has implications for the type of work the cobot can do, and where in the workflow the cobot can be inserted.

\section{DISCUSSION OF THE PRELIMINARY RE- SULTS}

Interestingly, the data set we obtained from our pilot study was much richer in terms of step-by-step procedures and detailed explanations of the reasons for doing a task in a particular way. Conducting the pilot study in our in-house workshop at the university provided the luxury of being able to probe deeply into the activity under scrutiny, whereas our presence on the live construction site was fitting in around the commercial building activity. We recorded what we could within the constraints of the time available onsite, coupled with the desire not to take up too much time of the builders whilst they were engaged in their normal activities. This meant that we could not stop and start as much, or ask them to repeat and explain what they were doing, as we could in the pilot study. In rethinking our methodology going forward, we consider it important to conduct observations in both types of context: the live construction site, and the workshop or lab.

When thinking about which parts of the human action repertoire observed on the building site are amenable to performance by a cobot, the question arises of whether the 'how' of performing the action is critical (See also Table 2). In some situations such as carrying a structure with a human co-worker, how the movement is performed could be critical, but in others such as bracing, it could be that the end result is the main criterion, not the motion path that is taken to execute it. Beyond physical motion analysis, simulation and transfer to robot manufacturing and construction, the social dimension of work is of significance, such as the role of talk amongst the carpenters which is critical for both checking and confirmation of performing tasks correctly and safely, but more importantly as social interaction to enrich work.

Given the amount of verbal and non-verbal communication that takes place on a building site, any cobot to be integrated into construction workflows will need a degree of fluency in understanding human speech and gesture. To make the shift from tool to partner in collaborative work, requires a cobot endowed with some form of human-machine intelligence. Otherwise, the robot remains in the category of tool, with the human in the role of operator. As with any new technology, the introduction of cobots into construction work will inevitably reshape current workflows and traditional practices of building. In our empirical observations of carpenters assembling timber frames for a house, the team of carpenters (or chippies) employed a range of agreed upon processes and routines, that were intimately linked with the materials and tools they used. Introducing a cobot into the already established working processes, as well as on-site, ad-hoc improvisations, would require a period of mutual learning and adaptation by the human carpenters.

\section{CONCLUSION AND OUTLOOK}

As initially discussed, machine learning has advanced to the point where industrial robots are able to be trained to learn how to perform a task, rather than current knowledge-based approaches. Consequently, after having established a methodology for workflow capture and analysis of carpentry tasks towards human-robot collaboration, the research team will investigate two pathways of training robots through machine learning - supervised and reinforcement learning. While a majority of existing research leverages reinforcement learning for training intelligence robots (Haarnoja \& Levine 2019 [2]; Levine et al. 2017; Ghadirzadeh et al. 2017) - a method that requires little to no training data - the research maintains the inclination to investigate both aforementioned forms of machine learning. This is congruous with a notion delineated by Pedros Domingos, who asserts that while reinforcement learning can be "vastly more powerful" than supervised learning, "requiring much less input knowledge to produce useful results, it still needs more than zero input knowledge to work" (2012, pp. 80). As next steps, the 
Table 2

An evaluation of the potential for insertion of a cobot in the workflow of collaborative actions identified in the activity of framing on a building site.

\begin{tabular}{|c|c|c|c|c|}
\hline & Type of cobot (arms/mobility) & & & \\
\hline Collaborative Action & 1-arm static & 2-arm static & 1-arm mobile & 2-arm mobile \\
\hline $\begin{array}{l}\text { 1. Sorting frame (lifting and moving to } \\
\text { pile) }\end{array}$ & No use, due to lack of mobility. & No use, due to lack of mobility. & $\begin{array}{l}\text { No use, as two arms are required to } \\
\text { hold and stabilise the frame. }\end{array}$ & $\begin{array}{l}\text { Possible, depending on relative size of } \\
\text { cobot to frame. }\end{array}$ \\
\hline 2. Carrying frames (2 carpenters) & No use, due to lack of mobility. & No use, due to lack of mobility. & $\begin{array}{l}\text { Can hold one end/side of } \\
\text { timber/structure with a grip end } \\
\text { effector, and locomote. }\end{array}$ & $\begin{array}{l}\text { Can hold one end/side of } \\
\text { timber/structure with two grip end } \\
\text { effectors, and locomote. }\end{array}$ \\
\hline 3. Sliding frames & No use, due to lack of mobility. & No use, due to lack of mobility. & $\begin{array}{l}\text { Can hold/push one end/side of } \\
\text { timber/structure with a grip end } \\
\text { effector }\end{array}$ & $\begin{array}{l}\text { Can hold/push one end/side of } \\
\text { timber/structure with two grip end } \\
\text { effectors }\end{array}$ \\
\hline 4. Rotating frames & No use, due to lack of mobility. & No use, due to lack of mobility. & $\begin{array}{l}\text { Can hold/lever one end/side of } \\
\text { timber/structure with a grip end } \\
\text { effector. May be limited by relative } \\
\text { size. }\end{array}$ & $\begin{array}{l}\text { Can hold/lever one end/side of } \\
\text { timber/structure with two grip end } \\
\text { effectors. May be limited by relative } \\
\text { size. }\end{array}$ \\
\hline 5. Foot tapping to location & $\begin{array}{l}\text { Can a robot arm replace the kicking } \\
\text { action of a human foot? }\end{array}$ & Ditto & Ditto & Ditto \\
\hline 6. Hammer tapping into position & $\begin{array}{l}\text { No use, due to lack of mobility and } \\
\text { one arm. }\end{array}$ & $\begin{array}{l}\text { Limited. } \\
\text { Only if the hammer tapping point is } \\
\text { where cobot is stationed. Assuming } \\
\text { one arm can stabilise the frame,whilst } \\
\text { the other arm hammer taps. }\end{array}$ & $\begin{array}{l}\text { Cobot can move to where hammer } \\
\text { tapping needs to be done, but has } \\
\text { only one arm. }\end{array}$ & $\begin{array}{l}\text { Cobot can move to where hammer } \\
\text { tapping needs to be done. One arm } \\
\text { can stabilise the frame, whilst the } \\
\text { other arm hammer taps. }\end{array}$ \\
\hline 8. Nailing frame to slab & $\begin{array}{l}\text { No use, due to lack of mobility and } \\
\text { one arm. }\end{array}$ & $\begin{array}{l}\text { Limited. } \\
\text { Only if the nailing point is where cobot } \\
\text { is stationed. Assuming one arm can } \\
\text { stabilise the frame,whilst the other arm } \\
\text { uses nail gun. }\end{array}$ & $\begin{array}{l}\text { Cobot can move to where nailing } \\
\text { needs to be done, but has only one } \\
\text { arm. }\end{array}$ & $\begin{array}{l}\text { Cobot can move to where tapping } \\
\text { nailing needs to be done. One arm } \\
\text { can stabilise the frame, whilst the } \\
\text { other arm uses the nail gun. }\end{array}$ \\
\hline
\end{tabular}

derived methodology can be adopted for two significant trajectories that will support a strategy of cobuilt as human-robot collaboration through supervised and reinforcement learning. This enables research teams and industry to understand the task or movement within its original context in which collaborative robots can assist humans on construction sites.

Supervised machine learning trajectory: The research team has started research into motion capture to act as the source of training data. Motion capturing of arm, hand, and finger movements while completing tasks will provide a data set that can be feed into machine learning and consequently train the robot to perform a movement or task. Motion capture has had a significant role in developing robot movement, as robots can learn from a human demonstrator (Stanton et al. 2012). A range of techniques for motion capture exist from limited technologies such as leap motion and kinect sensors to full body suits with incorporated data gloves that produce highly accurate results (Stanton et al. 2012). Exporting and processing the raw data, allows it to then be used for supervised machine learning to complete a desired movement or task within its range of movement (Stanton et al. 2012). However, as this trajectory is time and resource intensive, due to the need to capture each imaginable movement of a builder, the research investigates this trajectory mainly as a comparative study to evaluate and position the second trajectory the research aims to take.

Reinforcement learning trajectory: Whereas the supervised learning approach can be best thought of as learning to mimic actions through observation, reinforcement learning is more analogous to learning through trial and error. Through reinforcement learning, robots are tasked with achieving a given goal in whichever way they see fit. For this reason, they require no training data. Bansal et al. argue that due to the hands-off approach to training, reinforcement learning often uncovers emergent behaviours, originally unintended [1]. These behaviour are not necessarily undesirable. Rather than attempting to mimic human actions, the reinforcement learning approach focuses on mimicking human goals, potentially uncovering new approaches. With this trajectory, the 
data collected can be used as an objective measure for similarity.

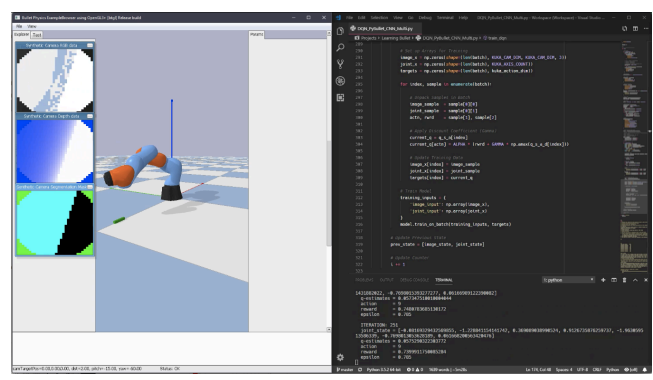

In conclusion, the contribution of this paper is the methodology for the empirical capture and analysis of human physical activity on a construction site. The methodology is grounded in interaction analysis, with a specific focus on the physical actions, movements, material and tool use, taking place in space and time, as well as a specific orientation to collaborative physical actions. Novel to our methodology is the set of visual resources generated from the interaction analysis, enabling identification and evaluation of potential insertion points for cobots in the already established workflows of construction workers. We illustrated the methodology in action through the case study of the framing process of carpenters on a building construction site. For the domain of construction, the methodology has potential to be applied across all the trades. In future work, the methodology will be extended to encompass the transition from the current output of the identification of actions suitable for cobots, to the aforementioned laboratory experimentation and testing of training the physical actions of cobots through machine learning.

\section{REFERENCES}

Brell-Cokcan, S and Braumann, J 2013, Rob | Arch 2012: Robotic Fabrication in Architecture, Art and Design, Springer Verlag, Wien

Colgate, JEC 1996 'Cobots: Robots for Collaboration with Human Operators', Proceedings of the International Mechanical Engineering Congress and Exhibition, At- lanta, pp. 433-439

Domingos, P 2012, 'A Few Useful Things to Know about Machine Learning', Cummun acm, 55(10), pp. 78-87

Ghadirzadeh, A, Maki, A, Kragic, D and Björkman, M 2017 'Deep predictive policy training using reinforcement learning', 2017 IEEE/RSJ International Conference on Intelligent Robots and Systems (IROS), pp. 2351-2358

Jordan, B and Henderson, A 1995, 'Interaction analysis Foundations and practice', The Journal of the Learning Sciences, 4, pp. 39-103

Levine, S, Pastor, P, Krizhevsky, A, Ibarz, J and Quillen, D 2018, 'Learning hand-eye coordination for robotic grasping with deep learning large-scale data collection', The International Journal of Robotics Research, 37(4-5), pp. 421-436

McKinsey, \& Company 2017, Reinventing Construction: A Route to Higher Productivity, McKinsey Global Institute

Rosenberg, E, Haeusler, M H, Araullo, R and Gardner, N 2015 'Smart Architecture区Bots and Industry 4.0 Principles for Architecture', 33rd eCAADE, Vienna, pp. 251-259

Stanton, C, Bogdanovych, A and Ratanasena, E 2012 'Teleoperation of a Humanoid robot using full-body motion capture, example movements, and machine learning', 012 Australasian Conference on Robotics and Automation (ACRA), pp. 260-269

Sylla, N 2017, Implementation of Collaborative Robot Applications, www.hssmi.org, no thesis type given, A Report from the Industrial Working Group

[1] https://ai.googleblog.com/2019/01/soft-actor-criticdeep-reinforcement.html

[2] https://openai.com/blog/competitive-self-play/

[3] www.sciencedaily.com/releases/2018/02/18020212

3732.htm

[4] https://www.technologyreview.com/s/541871/robo t-see-robot-do-how-robots-can-learn-new-tasks-by-obs erving/

[5] https://bim360resources.autodesk.com/robotics-inconstruction-jobsite/robotics-in-construction-a-brief-hi story-of-automation-in-the-industry

[6] https://theurbandeveloper.com/articles/australianbricklaying-robot-could-build-30-million-new-homes-g lobally-

[7] https://www.roboticvision.org/wp-content/uploads /Robotics-Roadmap_FULL-DOCUMENT.pdf
Figure 7

Screenshot of a simulation environment for the Kuka robot arm, that understands the mechanisms and data flow to control the arm, and weave a reinforcement learning algorithm in to interact with the simulation. 